\title{
High Performance RISC based 2D-DWT Architecture Implementation for Jpeg 2000
}

\author{
G. Lakshmi Prasanna \\ P.G.Scholar \\ Swarnandhra Institute of \\ Engg. \& Technology \\ Narsapur, A.P.
}

\author{
S. Ravi Chand \\ M.Tech (Ph.D.) \\ Department Of ECE \\ Swarnandhra Institute of \\ Engg \& Technology \\ Narsapur, A.P.
}

\begin{abstract}
In this paper a 2D-DWT architecture can be implemented using 5/3 lifting scheme. The 2D-DWT is used in many fields, they are signal analysis, computer vision, object recognition, image compression and video compression. The DWT is developed by the JPEG2000 which is the one of image compression standard based on the wavelet coding techniques.

The proposed architecture is RISC based architecture. The RISC processor has simple instruction set and gives high performance. The 5/3 lifting scheme is used to implement these architecture, which performs predict and update steps. The main objective of the project is to implement RISC based 2D-DWT architecture for processing images with high compression ratio's[i.e. limited size and better quality] compared to DCT which is one of the transform used in image compression.
\end{abstract}

\section{Keywords}

DWT, DCT, JPEG2000, RISC processor, 5/3 lifting.

\section{INTRODUCTION}

Digital imagery has had an enormous impact on industrial applications and scientific projects. It is no surprise that image coding has been a subject of great commercial interest in today's world. In many imaging applications, exact reproduction of the image bits is not necessary. An efficient transmission of data over a channel always remains a challenge as the speed of transmission and the clarity always affect each other. As the speed of transmission increases the bits transmitted has got higher tendency towards getting corrupted. This problem is much serious when comes to image transmission, as the images are represented in a large matrix dimension. Transmission of such big Matrices with a high speed always results in poor clarity on reception. To overcome this problem the image should be compressed before transmission.

Image compression is minimizing the size in bytes of a graphics file without degrading the quality of the image to an unacceptable level. The reduction in file size allows more images to be stored in a given amount of disk or memory

space. It also reduces the time required for images to be sent over the Internet or downloaded from Web pages.

Image transforms can be classified depends upon the nature of the basis functions as

1. Transforms with orthogonal basis function (FT, DCT and DST).

2. Transform with non-sinusoidal orthogonal basis functions (HAAR, WALSH, HADAMARD and SLANT).
3. Transforms whose basis functions depend on the statistics of the input data (KL and SVD).

4. Directional transformations (Hough, Radon, ridgelet, contourlet).

\section{IMAGE COMPRESSION}

Two ways of classifying compression techniques are mentioned below:

\section{Lossless Image compression \\ 2. Lossy Image compression}

In lossless compression schemes, the reconstructed image, after compression, is numerically identical to the original image. However, lossless compression can only achieve a modest amount of compression.

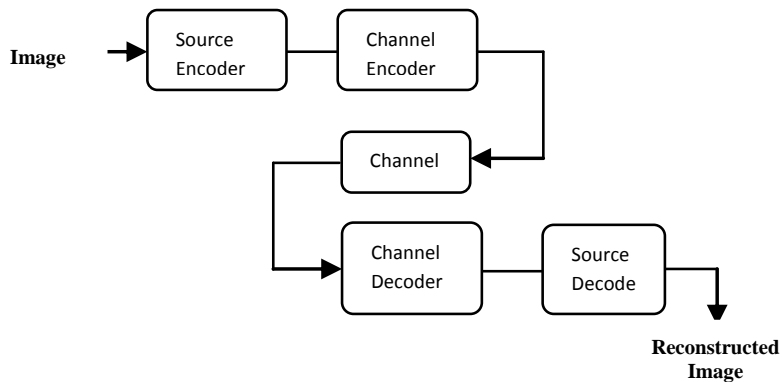

Fig.1.Block diagram of compression and reconstruction

An image reconstructed following lossy compression contain degradation relative to the original. Often this is because the compression scheme completely discards redundant Information. However, Lossy schemes are capable of achieving much higher compression under normal viewing conditions, no visible loss is perceived (visually lossless) under lossy compression.The compression and reconstruction of an image is as shown in fig. 1 .

\subsection{Genaral Lifting Scheme}

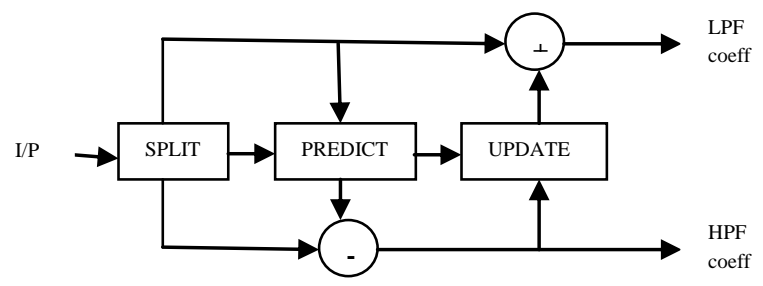

Fig.2.Block diagram of lifting scheme

The above fig.2. Shows the lifting scheme which consists of 3 steps, they are 


$$
\begin{array}{cl}
\text { i. } & \text { Split } \\
\text { ii. } & \text { Predict } \\
\text { iii. } & \text { update }
\end{array}
$$

1. Split: It is the first stage of the lifting scheme. It is used to separate the odd and even elements from $\mathrm{i} / \mathrm{p}$ data.

2. Predict: It is the second stage of lifting scheme. It uses a function that approximates the data set. It can replace the odd elements and even elements are unchanged and the even elements can be given to the $\mathrm{i} / \mathrm{p}$ for the next step.

3. Update: It is the third stage of the lifting scheme. The update stage follows the predict phase. It can replace the even elements.

\section{IMPLEMENTATION OF 2D-DWT ARCHITECTURE \\ 3.1 JPEG2000}

JPEG is a standardized image compression mechanism. JPEG stands for Joint Photographic Experts Group, the original name of the committee that wrote the standard. JPEG is designed for compressing full-color or gray-scale images of natural, real-world scenes. It works well on photographs, naturalistic artwork, and similar material; not so well on lettering, simple cartoons, or line drawings. JPEG handles only still images, but there is a related standard called MPEG for motion pictures.

"JPEG 2000" is a wavelet-based image compression standard. It was created by the JPEG (Joint Photographic Experts Group) committee with the intention of superseding their original discrete cosine transform-based JPEG standard.

JPEG 2000 can operate at higher compression ratios without generating the characteristic 'blocky and blurry' artifacts of the original DCT-based JPEG standard. It also allows more sophisticated progressive downloads.

\subsection{Image Compression Using Discrete Cosine Transforms}

The Discrete Cosine Transform is used in JPEG image compression standard. It is commonly used algorithm in image compression. It converts image pixels into sets of spatial frequencies. Discrete Cosine transform (DCT) is a mathematical function that transforms digital image data from the spatial domain to the frequency domain [1]. The DCT works by separating the images into parts of different frequencies [4].

The JPEG process is a widely used form of lossy image compression that centers around the DCT [6]. Compared to other transforms DCT decorrelate the image data. DCT has been widely used in signal processing of image data, especially in coding for compression, for its near optimal performance [1]. After de correlation each transform coefficient can be encoded independently without loosing compression efficiency [6].

Some properties of the DCT which are of particular value to image processing applications [6]:
1. De-correlation.
2. Energy compaction.
3. Separability.

4. Symmetry.

5. Orthogonality.

\subsection{Image Compression Using Discrete Wavelet Transforms}

The term wavelet comes from the fact that they integrate to zero; they wave up and down across the axis [3].Discrete Wavelet Transform (DWT) can be efficiently used in image coding applications because of their data reduction capabilities [5]. The DWT has been introduced as a highly efficient and flexible method for sub-band decomposition of signals [3]. Unlike the case of Discrete Cosine Transform (DCT) which basis is composed of cosine functions, basis of DWT can be composed of any function (wavelet) that satisfies requirements of multi-resolution analysis [5]. DWT is used as basis for transformation in JPEG 2000 standard and DWT provides high quality compression at low bit rates [7].

The below fig.3. Shows the forward 1D-DWT used for compress the image. The original image is passed through high pass and low pass filter.

The fig.4. Shows the forward 2D-DWT which can use set of low pass and high pass filters. The original data is passed from both low and high pass filters, both the outputs are down sampled by 2 .

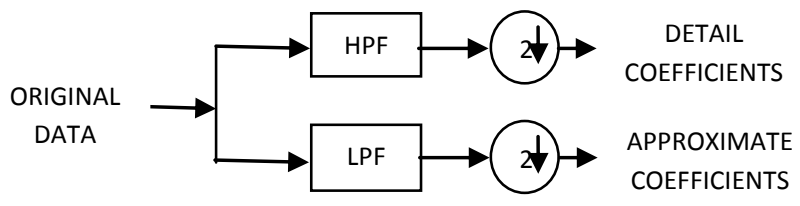

Fig3.Block diagram of 1-D DWT

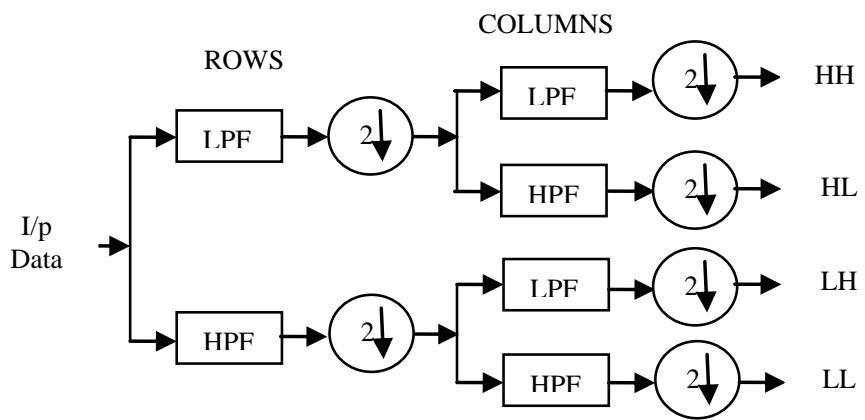

Fig4. Forward 2D-DWT

It performs row and column operations. The data first processed in row and then processed in column. It gives four output coefficients as LL, HL, LH, HH. All the outputs of LPF and HPF are down sampled by 2. The horizontal elements are $\mathrm{LH}$, vertical elements are HL and diagonal elements are $\mathrm{HH}$ respectively. 


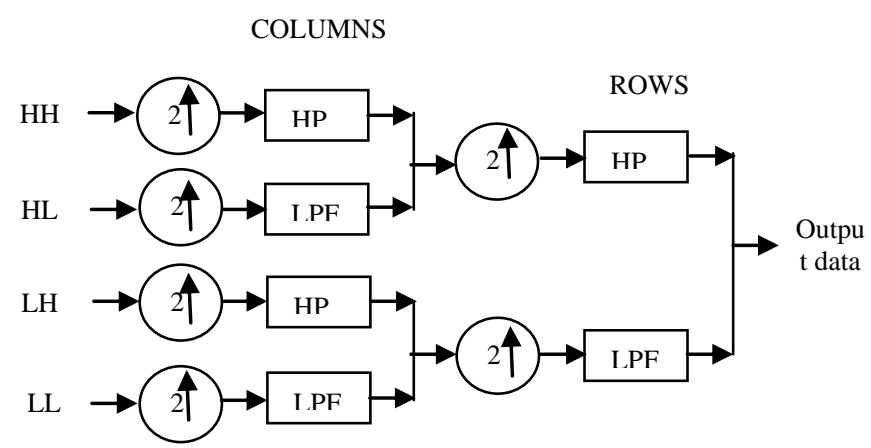

Fig.5.Inverse 2D-DWT

The fig.5. Shows the inverse 2D-DWT used for reconstruct the image. It is also designed using set of LPF and HPF filters. The four compressed outputs [i.e., four coefficients LL, HL, $\mathrm{LH}$ and $\mathrm{HH}]$ are given to the inputs of inverse 2D-DWT. The coefficients are up sampled by factor 2 . It transform first column and then transform row.

\subsection{Risc Processor}

RISC processor means reduced instruction set computing. It has simplified instruction set and give high performance. Only load and store instructions are accessed by memory with simple, single addressing modes. The instructions have a fixed size and few simple formats. It can use pipelining for implementing architecture and their instruction set allow tight control of their pipeline by the software.

The advantages of RISC processor is,

1. High performance at low cost

2. Higher clock rates are allowed by smaller and simpler circuits

3. The data path can use pipelining and the compiler can exploit it.

4. It can reduce the design time, cost and silicon area.

\section{2-D DWT SYSTEM ARCHITECTURE}

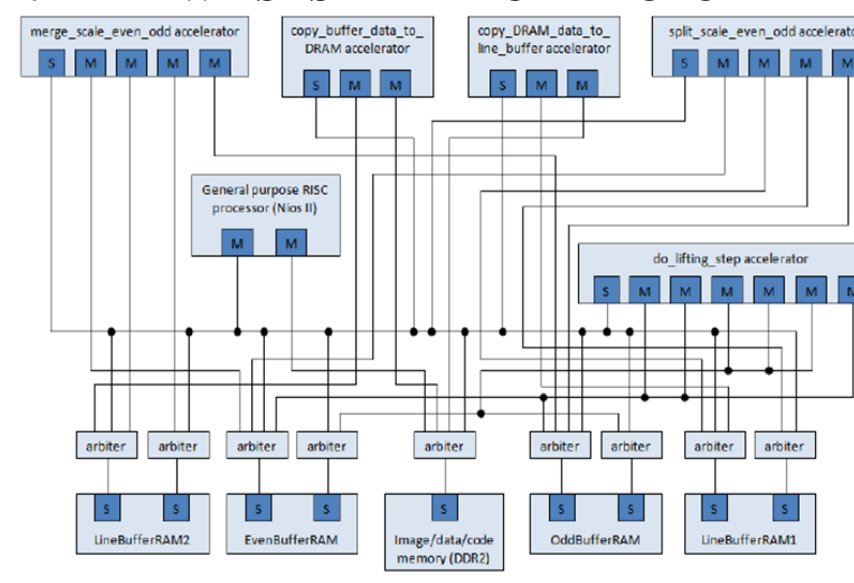

Fig.6.Architecture of 2D-DWT

The fig. 6 shows the 2D-DWT system architecture using RISC processor. In this 5 hardware accelerators and memory buffers are interconnected using master-slave interfaces.

These hardware accelerators perform either forward or inverse operations and these can be controlled by master CPU. To use lifting filters depending upon the applications.
The main computations are performed by the accelerators are split_scale_even_odd, do_lifting_step and merge_scale_even_odd for 2D-DWT processing. The DMA mechanism is performed by the two accelerators are copy_DRAM_data_to_line_buffer and copy_buffer_data_to_DRAM are used in combination with master CPU. The DMA transfer of 32-bit words from DRAM to 16-bit half word dual ported line buffer memory is the destination and vice versa.

Table 1. Integer approximation values used for fixed point Implementation of the jpeg2000 filters.

\begin{tabular}{|c|c|c|c|}
\hline \multirow{4}{*}{$\begin{array}{l}\text { Filter } \\
\begin{array}{l}\text { CDF 9/7 } \\
\text { filter }\end{array}\end{array}$} & \multicolumn{2}{|c|}{ Lifting factor } & \multirow{2}{*}{$\begin{array}{c}\begin{array}{c}\text { Integer values of } \mathbf{~ m} \\
\text { used for rounding } \\
(\mathbf{s}=\mathbf{1 3})\end{array} \\
25987\end{array}$} \\
\hline & $\mathrm{a}$ & 1.58613 & \\
\hline & $\mathrm{b}$ & 0.05298 & 868 \\
\hline & $\mathrm{c}$ & 0.88291 & 14466 \\
\hline & d & 0.44351 & 7266 \\
\hline & $\mathrm{k}$ & 1.14960 & 18835 \\
\hline & $1 / \mathrm{k}$ & 0.86986 & 14252 \\
\hline LeGall & $\mathrm{a}$ & 0.5 & 8192 \\
\hline $5 / 3$ filter & $\mathrm{d}$ & 0.25 & 4096 \\
\hline & $\mathrm{k}$ & 1.41423 & 23171 \\
\hline & $1 / \mathrm{k}$ & 0.70711 & 11585 \\
\hline
\end{tabular}

\subsection{2-D Dwt System Architecture Algorithm}

1. This algorithm shows how DWT processing is carried out in the proposal architecture.

2. The $5 / 3$ lifting filter is used to perform the forward and inverse DWT respectively.

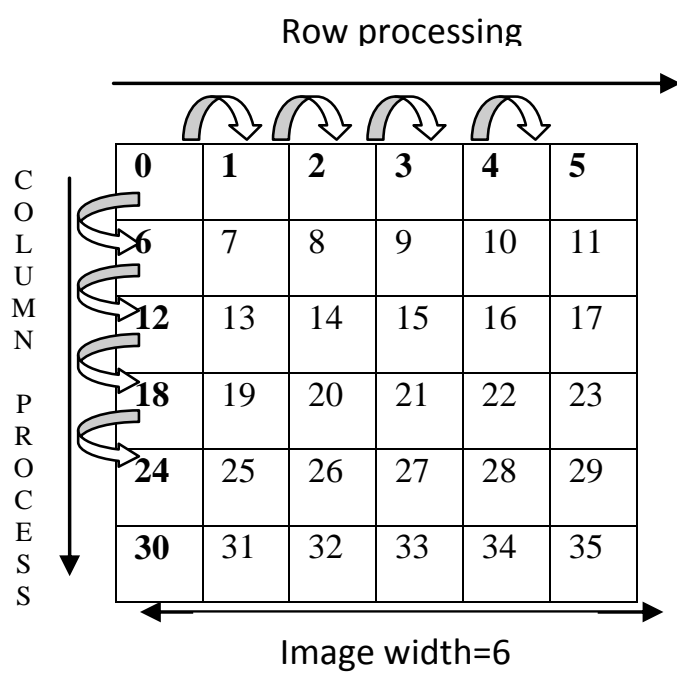

Fig.7.row/column processing of 2D-data

3. The $5 / 3$ lifting scheme use three hardware accelerators which can perform the main computations of 2D-DWT.

4. The integer values of $m$ are haven in table 1 are passed to these three accelerators to determine the lifting factors as required by the selected DWT lifting mode. The lifting factors are tabulated in table no. 1

5. The first stage is the split_scale even odd accelerator which separates the even and odd data from line buffer RAM1 memory they can stored in even and odd buffer RAM memory locations. 
6. The second stage is the do_lifting_step accelerator which performs predict and update modes.

7. The final stage of DWT processing is merge_scale_even_odd accelerator.

\subsection{Implementation}

\section{Row/Column processing of 2D Data}

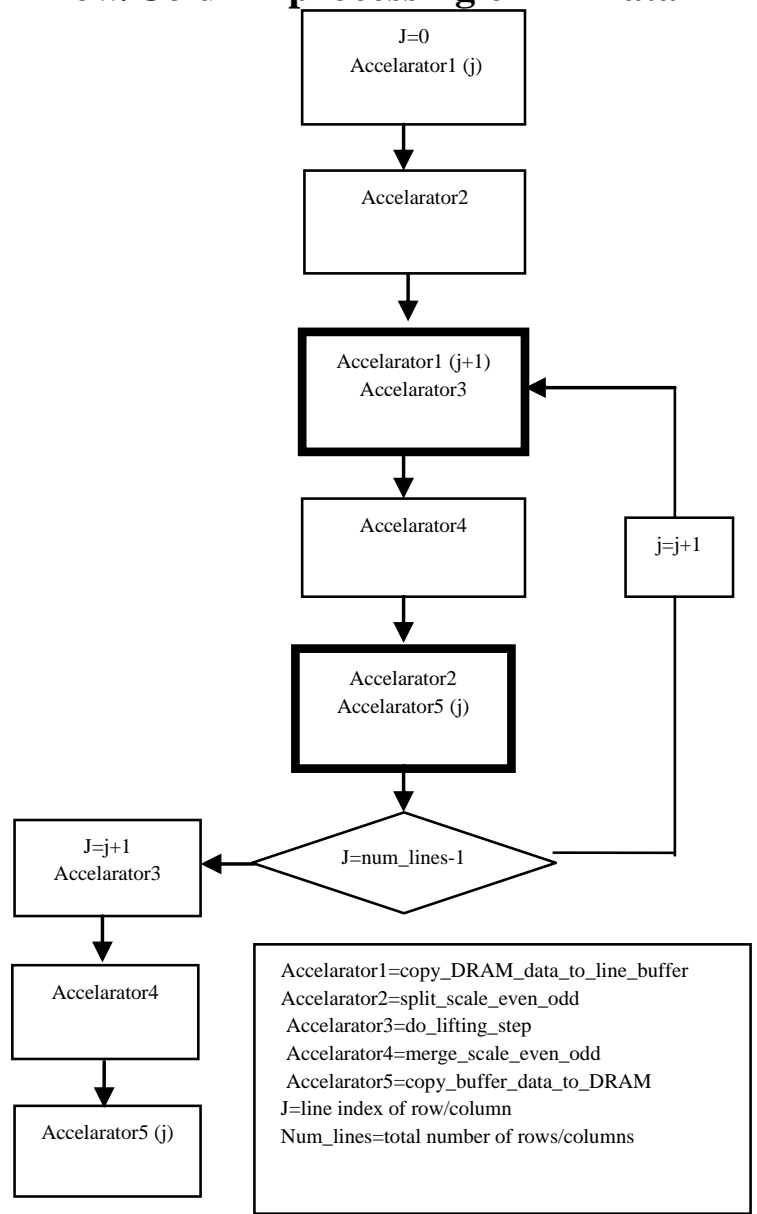

Fig.8.flow chart showing row/column processing of 2D data.

1. The fig7 shows the implementation of $2 \mathrm{D}$ data processing.

2. The combination of two hardware accelerators [i.e.copy_DRAM_data_to_line_buffer and copy_line_buffer_data_to_DRAM] and master CPU perform the row and column processing.

3. The highlighted pixels in fig.7 represent the line index register values passed to the two hardware accelerators to determine row/column processing.

4. The flowchart shown in fig8 demonstrates all accelerators can work simultaneously.

5. The row/column processing of $2 \mathrm{D}$ data represented in the highlighted boxes of the flowchart in fig7.

6. The even and odd indexed data stored in even and odd buffer RAM respectively.

7. The input line data stored in line buffer RAM1memory.
8. Next row/column of the image can prefetch by the copy_DRAM_data_to_line_buffer into line buffer RAM1.

9. The output coefficients are stored in line buffer RAM2 memory.

10. The copy buffer data to DRAM accelerator transfers the output coefficients into original image.

11. The split_scale_even_odd accelerator processes the next input line data stored in line buffer RAM1 memory.

\section{COMPARISION BETWEEN DWT AND DCT}

Various parameter comparison of DCT Vs DWT is tabulated in table2.

Table no.2 comparison of DCT Vs DWT

\begin{tabular}{|l|l|l|}
\hline Parameter & DCT & DWT \\
\hline Compression ratio & 20.1763 & 20.3955 \\
\hline PSNR & 0.0263 & 38.6309 \\
\hline MSE & 10.3820 & 8.9123 \\
\hline BER & 37.9680 & 0.0259 \\
\hline TIME & 7.4121 & 3.5139 \\
\hline
\end{tabular}

The DWT compared with DCT, DWT has

1. Higher compression ratios and avoids blocking artifacts.

2. Transmission time is less.

3. DWT allows good localization both in spatial and frequency domain. In this image introduces inherent scaling.

\section{RESULT AND CONCLUSION}

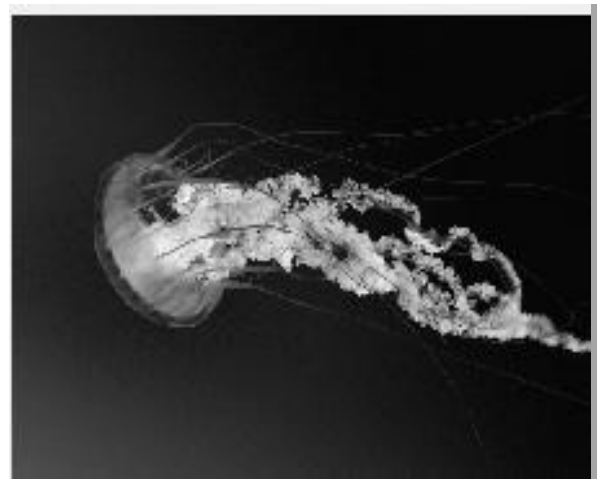

Fig.9.Original image

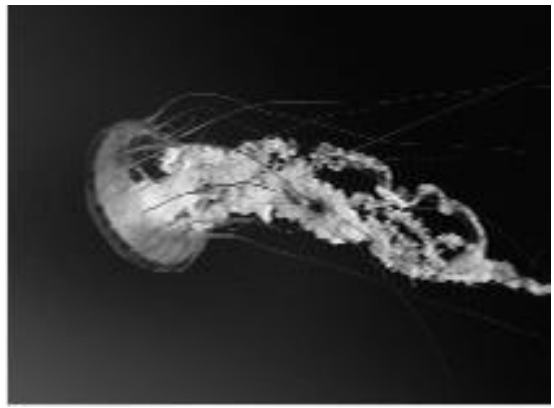

Fig.10.DCT compressed image 


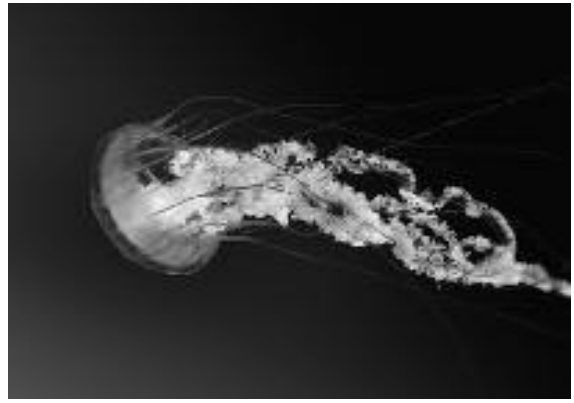

Fig.11.DWT compressed image

In this paper 2D-DWT architecture is implemented using RISC processor and also compared it with other compression standards. The RISC based 2D-DWT architecture consists of five hardware accelerators; each accelerator is co-ordinate by a master CPU to perform different operations for processing 2D data. This is highly programmable and scalable in performance. It gives better image quality and limited size capability and also compared with DCT.

\section{REFERENCES}

[1] Nageswara Rao Thota1, Srinivasa Kumar Devireddy2 "Image Compression Using Discrete Cosine Transform"

[2] M. MozammelHoque Chowdhury and Amina Khatun "Image Compression Using Discrete Wavelet Volume Transform" IJCSI International Journal of Computer Science Issues, Vol. 9, Issue 4, No 1, July 2012 ISSN (Online): 1694-0814
[3] Dipalee Gupta1, Siddhartha Choubey2 "DISCRETE WAVELET TRANSFORM FOR IMAGE PROCESSING'International Journal of Emerging Technology and Advanced Engineering Website: www.ijetae.com (ISSN 2250-2459, ISO 9001:2008 Certified Journal, Volume 4, Issue 3, March 201.5

[4] Amanjot Kaur 1, Jaspreet Kaur "Comparison of DCT and DWT of Image Compression Techniques" International Journal of Engineering Research and Development ISSN: 2278-067X, 1, Issue 4 (June 2012).

[5] Monika Rathee, AlkaVij "Image compression Using Discrete Haar Wavelet Transforms" ISO 9001:2008 Certified International Journal of Engineering and Innovative Technology (IJEIT) Volume 3, Issue 12, June 2014.

[6] Syed Ali Khayam "The Discrete Cosine Transform (DCT): Theory and Application1" Department of Electrical \& Computer Engineering Michigan State University March 10th 2003.

[7] Bhawna Gautam "IMAGE COMPRESSION USING DISCRETE COSINE TRANSFORM \& DISCRETE WAVELET TRANSFORM" National Institute of Technology, Rourkela May, 2010.

[8] BhondeNilesh, Shinde Sachin, NagmodePradip, D.B. Rane, "Image Compression Using Discrete Wavelet Transform". International Journal of Computer Technology and Electronics Engineering (IJCTEE) Volume 3, Special Issue, March-April 2013, An ISO 9001: 2008 Certified Journal. 